\title{
Neutron scattering on magnetic nano-objects
}

\author{
Frédéric Ott
}

Laboratoire Léon Brillouin, UMR12 CEA-CNRS, Centre d'Etudes de Saclay, 91191 Gif-sur-Yvette, France

\section{MAGNETIC NANOSTRUCTURES}

During the last 20 years, the field of the magnetism of nanostructures has exploded. A huge number of new magnetic structures have appeared in which the nanometer scale plays a key role. It is possible to classify them into 3 categories:

- In 3 dimensions, it is possible to identify a wide range of systems such as (i) nanoparticles in solutions forming ferrofluids, (ii) percolating phases as found in manganites for example, (iii) magnetic domains or domain walls in crystals, (iv) long range helical structures as found in $\mathrm{MnSi}$ or $\mathrm{BiFeO}_{3}$ for example. These objects are ideally studied using Small Angle Neutron Scattering (SANS) which enable measuring either the shape of individual objects (form factor) or their long range organization (structure factor).

- In 2 dimensions, nanometer size objects organized on surfaces. These objects can be either produced by lithography techniques or by self-organization processes. The scattering technique which can be used is either SANS or Grazing Incidence SANS.

- In 1 dimension, thin films produced by physical means such as vacuum deposition (sputtering, evaporation, laser ablation ...): metal thin films, oxide thin films, magnetic semi-conductors. These systems are most ideally studied by polarized neutron reflectivity. This is the object of a dedicated chapter.

In this chapter we describe in a first part the basics of SANS scattering applied to the study of magnetic nano-objects. This is illustrated by a few examples of recent studies on magnetic nanoparticles and phase separated materials. In a second part we discuss the GISANS technique and illustrate its use in the case of the study of self-organized magnetic domains.

\section{SMALL ANGLE NEUTRON SCATTERING}

One of the most mainstream technique used in neutron scattering is Small Angle Scattering. It is mostly used for polymer science and soft matter studies because of the contrast variation possibilities. It can also be used to perform studies on magnetic materials and take benefit of the strong magnetic scattering. This allows to probe nanometric properties of magnetic crystals.

This is an Open Access article distributed under the terms of the Creative Commons Attribution License 4.0, which permits unrestricted use, distribution, and reproduction in any medium, provided the original work is properly cited. 


\section{Collection SFN}

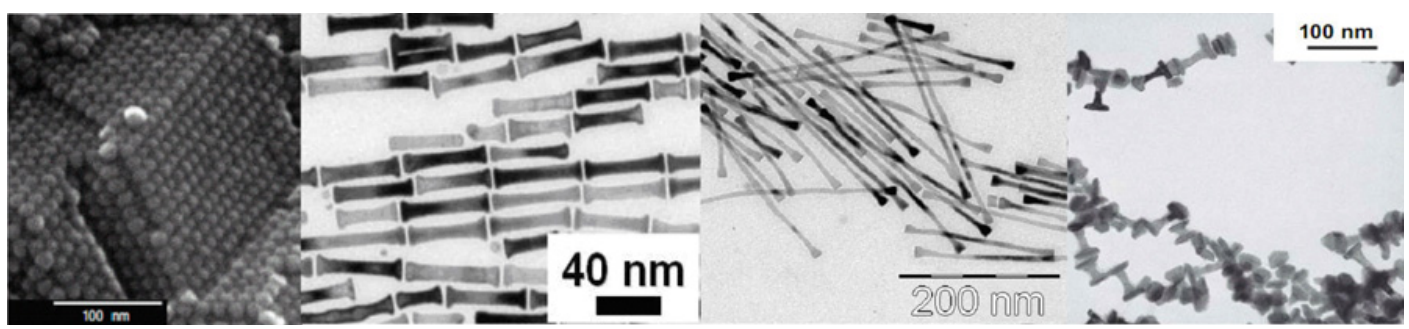

Figure 1. (Left) crystal of FeCo nanoparticles [1]; (right) $\mathrm{Co}$ and $\mathrm{Co}_{80} N i_{20}$ nanowires ([2]).

\subsection{Principles}

The neutron-matter interaction potential is given by two main contributions, the neutron/nucleus and the neutron/magnetic induction interactions:

$$
V=\frac{2 \pi \hbar^{2}}{m} b_{n} \delta(r) \text { and } V_{M}=-\mu \cdot B(r) .
$$

Since we are considering scattering at small scattering wave-vectors, we are not sensitive to the atomic details of the matter and the optical approximation can be applied. It is thus possible to define a scattering length density as:

$$
b_{v o l}=\frac{1}{V} \sum b_{i}
$$

where the volume $V$ over which the average is taken is of the order of a few $\mathrm{nm}^{3}$.

If one considers nano-objects in a matrix (Fig. 3), SANS measures the form factor of the object which is the Fourier transform of the scattering length density contrast between the scattering object and its matrix $\Delta b=b_{1}-b_{0}$. The measured intensity is the form factor squared:

$$
I(Q)=|F T(\Delta b(r))|^{2}=|F(Q)|^{2} .
$$

For the usual wavelengths and collimations used on small angle scattering spectrometers, the accessible $Q$ range is $0.02-2 \mathrm{~nm}^{-1}$ which corresponds to lengths in real space ranging from 3 to $300 \mathrm{~nm}$.

In the case of magnetic systems, the interaction is limited to the component of the magnetization perpendicular to the scattering wave-vector $Q$. The interaction potential has thus the form $F_{M}(Q) \sin (\alpha)$ where $F_{M}=F T\left(\triangle b_{m}\right)$ is the magnetic form factor and $\alpha$ is the angle between the magnetization and the scattering wave-vector.

With polarized neutrons one can measure two scattered intensities $I^{+}$and $I^{-}$depending on the orientation of the neutrons polarization with respect to the applied field:

$$
\begin{aligned}
& I^{+}(Q)=\left|F_{N}+F_{M} \sin \alpha\right|^{2} \\
& I^{+}(Q)=\left|F_{N}-F_{M} \sin \alpha\right|^{2} .
\end{aligned}
$$

These intensities can be combined to provide the following relations:

$$
\begin{gathered}
\left(I^{+}+I^{-}\right) / 2=F_{N}^{2}+F_{M}^{2} \sin ^{2} \alpha \\
I^{+}-I^{-}=4 F_{N} F_{M} \sin \alpha .
\end{gathered}
$$

When performing a polarized SANS measurement, it is possible to consider 2 geometries. The first possibility is to apply a field parallel to the neutron propagation direction (Fig. 4a). If in this situation the magnetization is parallel to the applied field, the scattering will be isotropic. The second possibility 

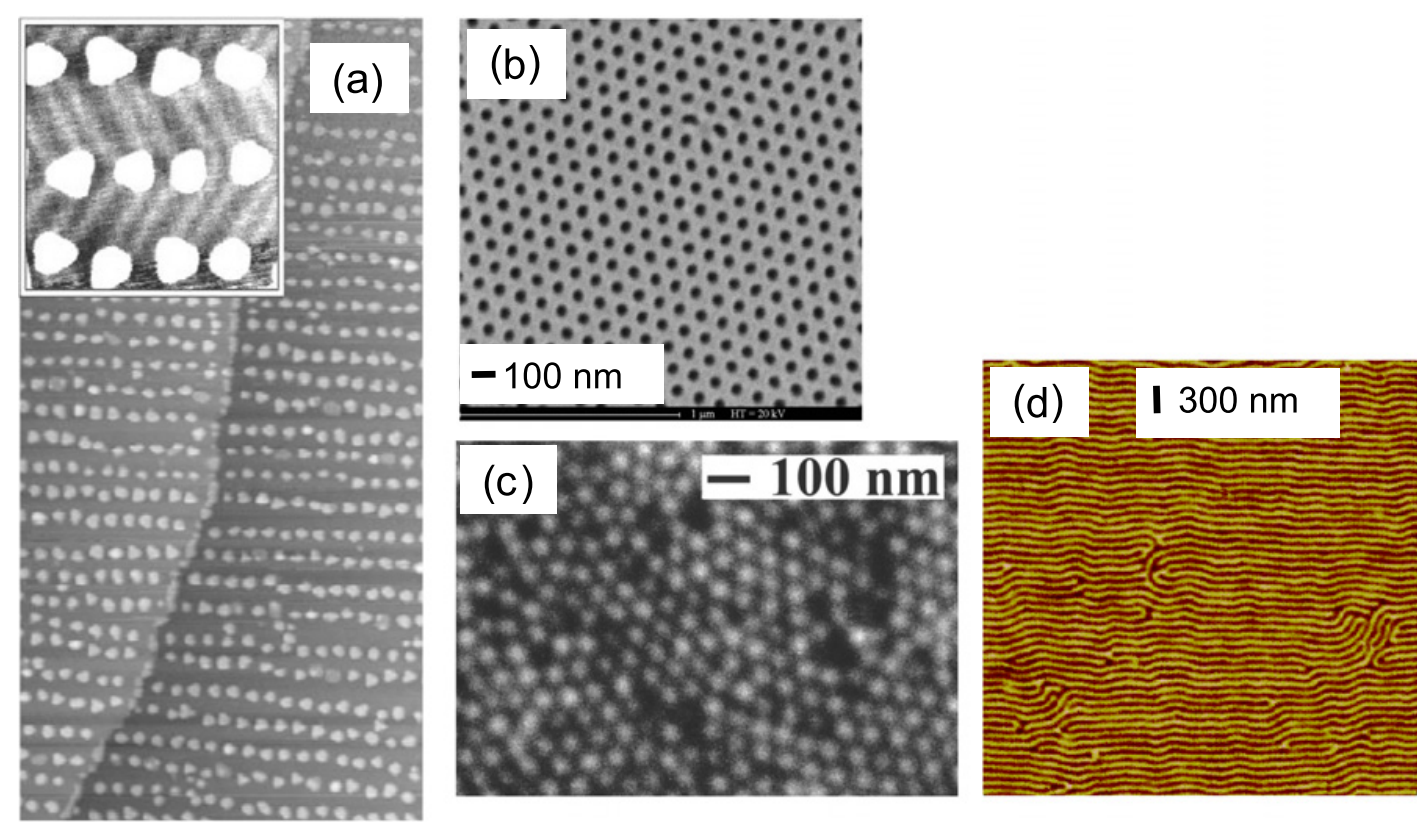

Figure 2. (a) $300 \times 300 \mathrm{~nm}$ image of self-organized Co dots on a gold substrate [3]. (b) Alumina membrane with pores of diameter $60 \mathrm{~nm}$ in which metals can be electrochemically grown. (c) Fe nanodots with average diameter and periodicity of 32 and $63 \mathrm{~nm}$ grown using a $\mathrm{Al}_{2} \mathrm{O}_{3}$ mask [4]. (d) Magnetic domains in a FePt thin film. The periodicity of the pattern is $100 \mathrm{~nm}$.

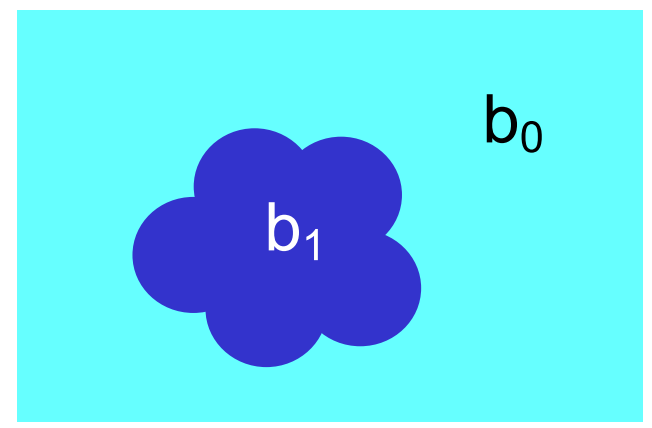

Figure 3. Object of scattering length $b_{1}$ in a matrix or solvent of scattering length $b_{0}$.

is to apply a magnetic field perpendicular to the neutron propagation direction (Fig. 4b). In this case, the scattered intensity is modulated by $\sin ^{2} \alpha$ factor (Fig. 4c). The magnetic scattered intensity is maximum in the direction perpendicular to the applied field $\left(\alpha=90^{\circ}\right)$.

Some of the key advantages of SANS are that: (i) there is a strong scattering difference between hydrogen and deuterium which allows to perform selective labeling (of surfactants for example); (ii) the magnetic scattering is very large which makes quantitative measurements possible; (iii) Neutrons are barely absorbed, which permits to look at bulk samples and to use complex sample environments (low temperatures, high magnetic fields).

Figure 5 gives a sketch of a typical Polarized SANS instrument on a neutron reactor source. 


\section{Collection SFN}
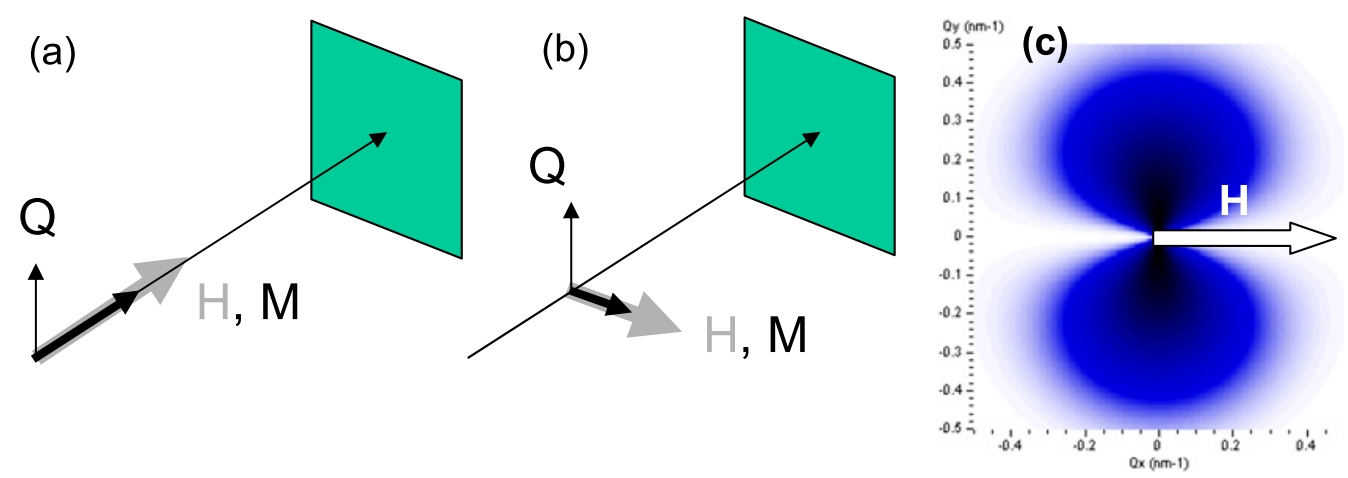

Figure 4. Different configurations for polarized small angle scattering. The field can be applied longitudinal (a) or

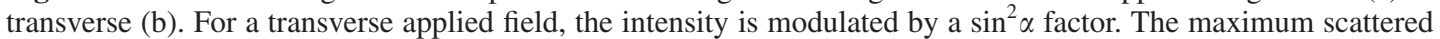
intensity is obtained in the direction perpendicular to the field (c).

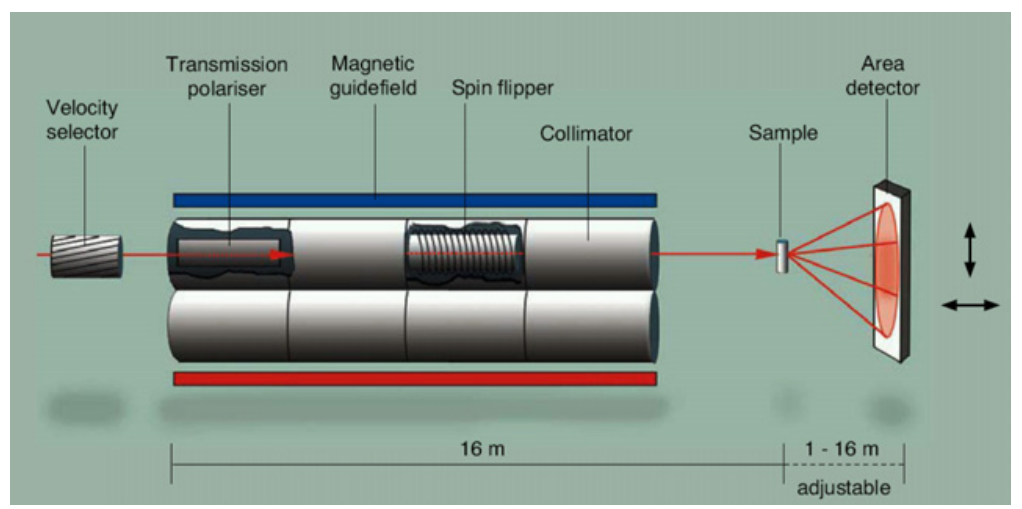

Figure 5. PSANS instrument V4 at BENSC (HMI Berlin).

\subsection{Magnetic nanoparticles}

Let us consider nano-spheres in a solvant (Fig. 6a). Their Scattering Length Density is either high or lower than the SLD of the solvant (Fig. 6b). The form factor of a sphere can be calculated analytically and is given by

$$
P(Q)=\left(\frac{3}{(Q R)^{3}}(\sin (Q R)-Q R \cos (Q R))\right) .
$$

In the case where the system is isotropic, the 2D signal can be integrated circularly in order to provide a more readable view of the signal (Fig. 6d). When the size distribution of the system is small, one observes well defined oscillations (black line on Fig. 6d). When the nano-objects exhibit a size dispersion, the oscillations are smoothed (red line on Fig. 6d).

The above example dealt with the ideal case of perfect spheres without internal structure. In practice, the magnetic nano-objects may exhibit a much more complex structure (Fig. 7). Magnetic nanoparticles may be constituted of a magnetic core, a non magnetic shell (due to oxidation for example) and quite often of a surfactant shell which allows the particle to remain in suspension in the solvant. Using SANS, it is possible to study in details the structure of such particles. Information such as the diameter of the magnetic core, the thickness of the non magnetic shell and the thickness of the surfactant may be 

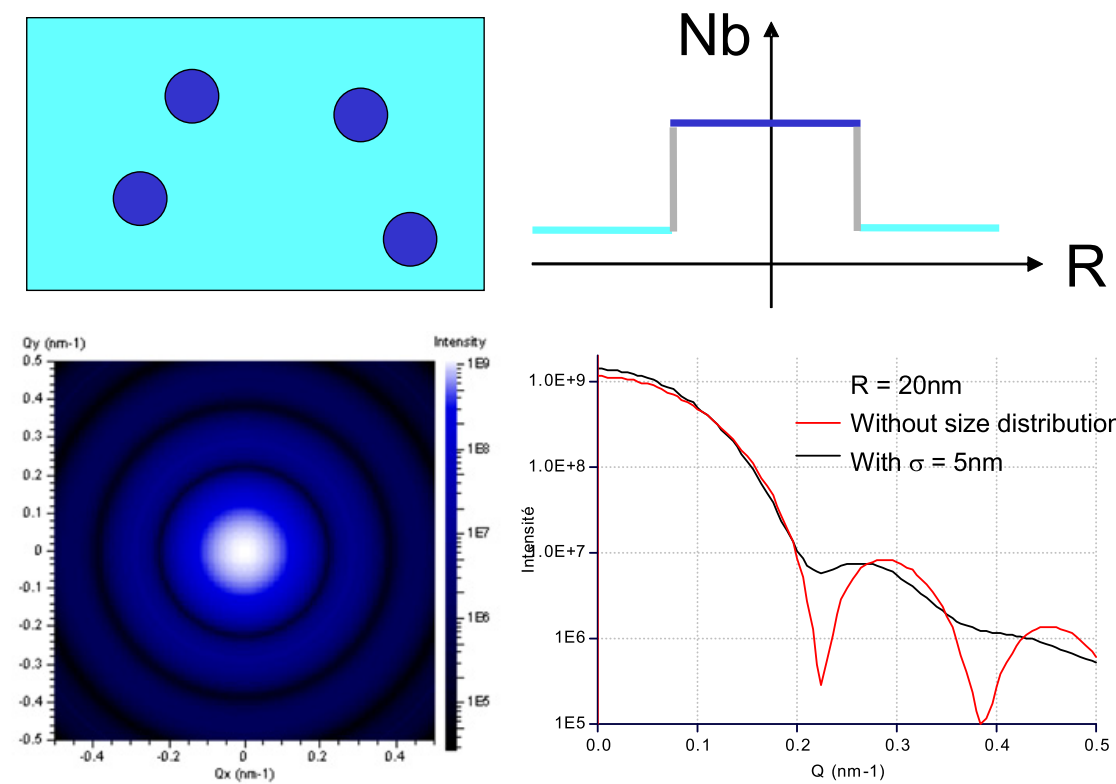

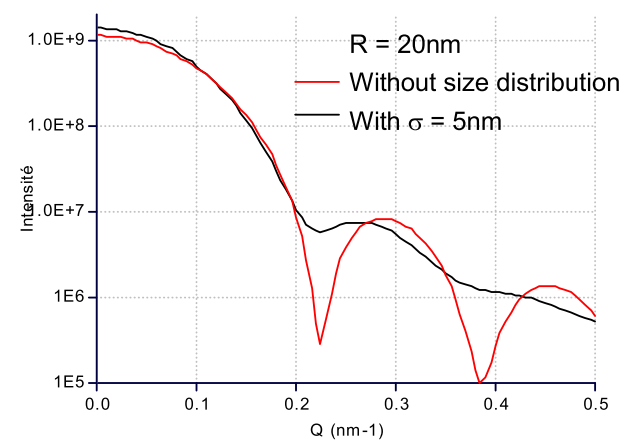

Figure 6. Form factor of a sphere in a solvant. (a) colloidal suspension of spheres; (b) their scattering length density in the liquid; (c) the scattering form factor; (d) the circularly integrated form factor (with and without size distribution).

measured using a combination of Polarized SANS measurements and the contrast variation method which allows varying the contrast of the organic shell with respect to the solvant. This contrast can be highlighted or extinguished by varying the Scattering Length Density of the solvant. In the case of water $\left(\mathrm{H}_{2} \mathrm{O}-\mathrm{D}_{2} \mathrm{O}\right)$, the SLD can be varied continuously from $\left(-5.6\right.$ to $\left.+64 \times 10^{13} \mathrm{~m}^{-2}\right)$.

We illustrate this point in the case of oxidized Co particles coated with a surfactant in colloidal suspension in a toluene solution (Fig. 7). Polarized SANS measurements in H and D-toluene solvent can provide the layered structure of these particles with great details [5] (Fig. 7).

\subsection{Structure factors}

In the case of dense packing of nano-objects, the scattering becomes sensitive to the position correlation between the particles. The measured intensity is given by:

$$
I(Q)=|F(Q)|^{2} \cdot S(Q)
$$

where $S(Q)$ is the structure factor which characterizes the correlations between the particle positions.

The system can be more or less well packed which leads to structure factors which are more or less well defined (see Fig. 8). In practice, it is difficult to observe any correlation peaks in the structure factor beyond the second nearest neighbour.

We can illustrate the effect of dense packing of nanoparticles on SANS patterns in the case of an assembly of Co nanoparticles. Figure 9 (top) shows the ordering of magnetite nanoparticles on a surface under a ZFC and FC procedure; Figure 9 (bottom) shows the ordering of magnetite nanoparticles in bulk under ZFC and FC procedures [6].

In the above summary, we have presented examples of small angle scattering on well defined objects such as nano-spheres. More generally, it is possible to perform SANS studies to characterize magnetic 


\section{Collection SFN}

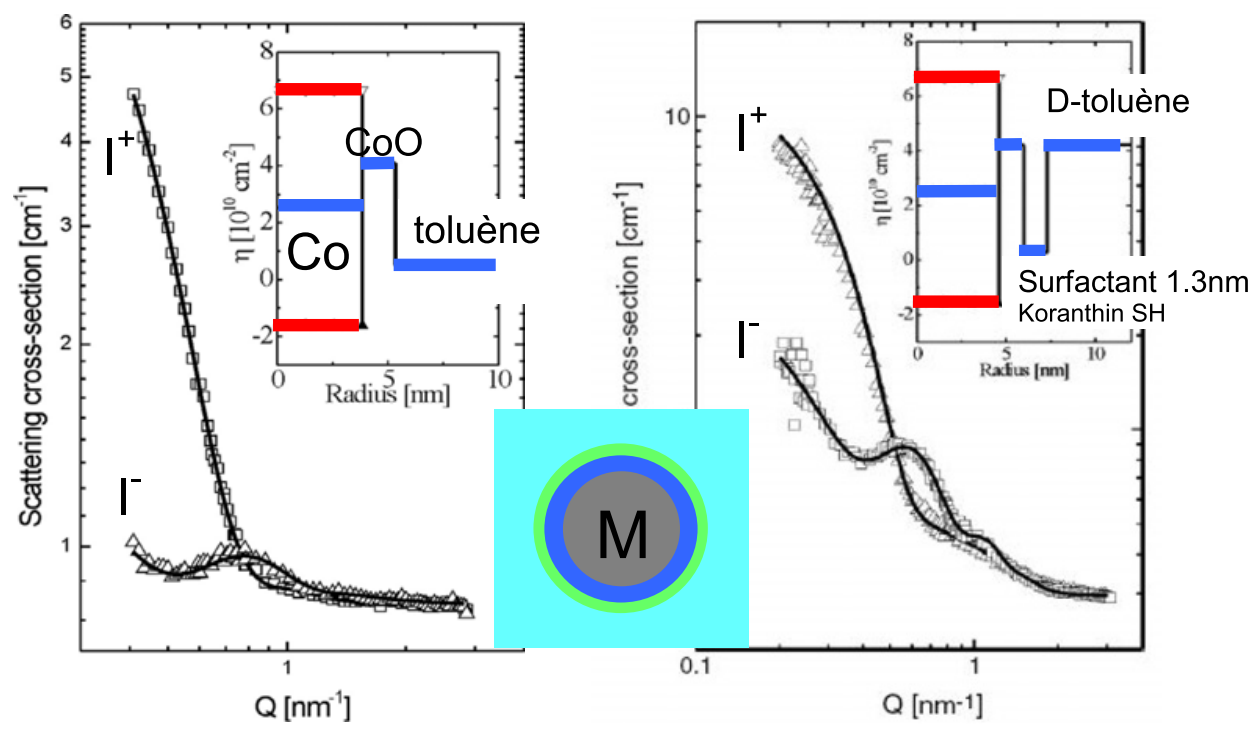

Figure 7. Magnetic Co particle with a magnetic core (gray), an oxidized surface (blue), a surfactant layer (green), floating in solvent (blue). It is possible to determine the thickness and the scattering lengths of these different layers by PSANS. (Adapted from A. Wiedenmann et al [5].)
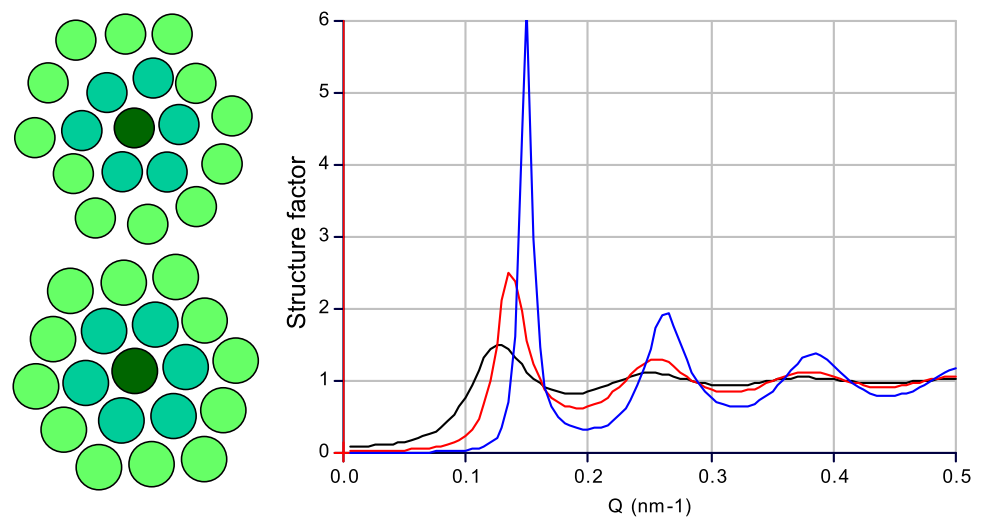

Figure 8. Dense packing of particles. The system can be more or less well packed which leads to different structure factors (packing density of $0.3,0.45,0.6$ ) in a Percus-Yevick approximation.

critical scattering [8], the penetration of magnetic flux in superconductors [9], or any other type of magnetic correlations taking place at a nanometric scale.

The study of the magnetic properties of solid materials is much better performed on single crystals. Past experiences have shown that the use of powders led to very large parasitic SANS signals. In order to perform clean studies, it is thus necessary to use single crystals. This is not necessarily a limitation since rather small crystals are required $\left(\mathrm{a} \mathrm{few} \mathrm{mm}^{3}\right.$ ). In the following section, we present an example of study of a nanometric phase separation in a magnetic single crystal. 

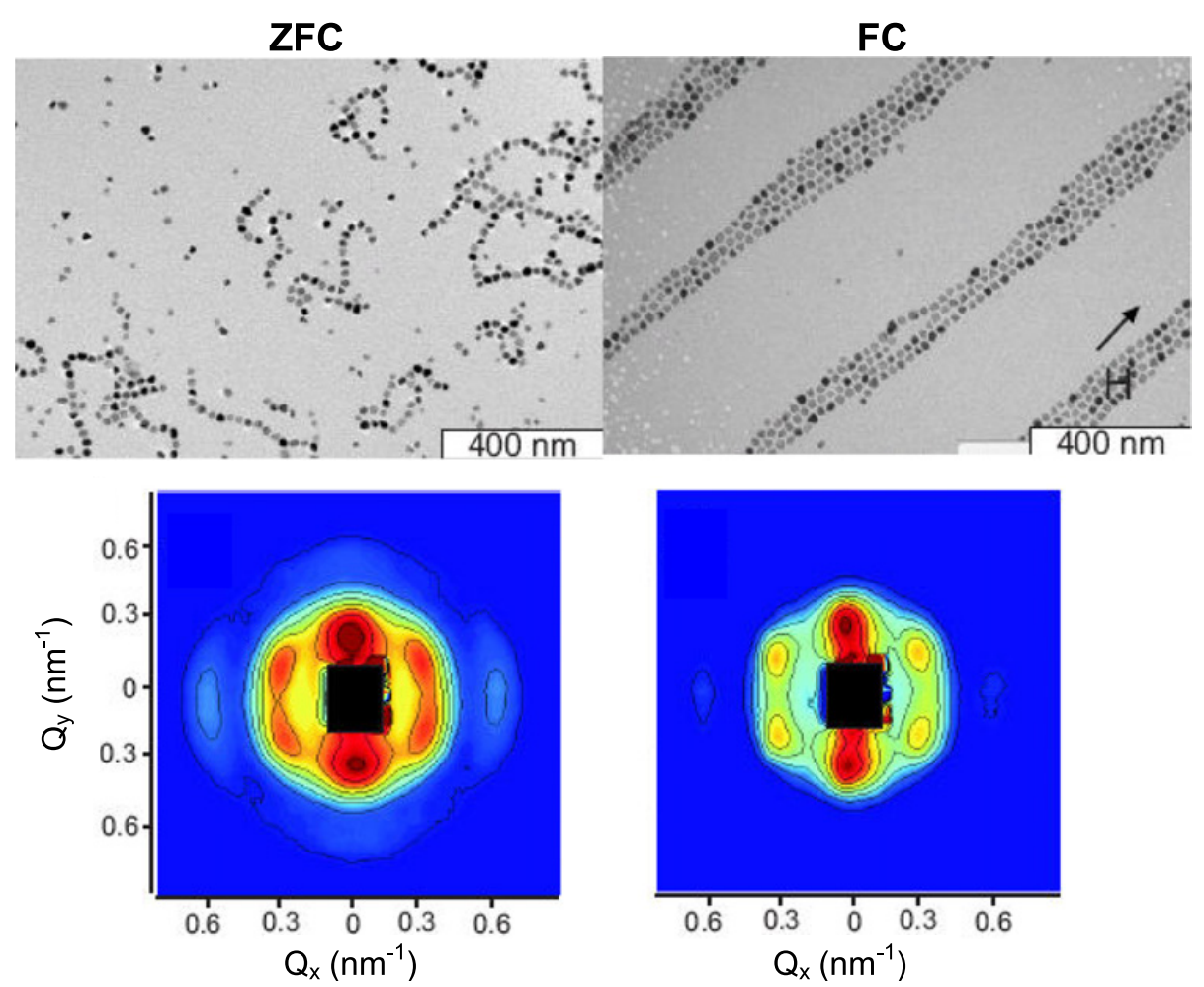

Figure 9. (Top) ordering of magnetite nanoparticles on a surface with a ZFC and FC procedure; (bottom) ordering of magnetite nanoparticles in bulk in ZFC and FC procedure (adapted from [6]). Under field, a well-defined hexagonal array forms.

\section{MAGNETIC FILAMENTS IN $\operatorname{Pr}_{0.67} \mathrm{Ca}_{0.33} \mathrm{MnO}_{3}$ CRYSTALS - HOPPING EXCHANGE}

Manganites $\left(\mathrm{A}_{x} \mathrm{~B}_{1-x} \mathrm{MnO}_{3}\right.$ ) (where $\mathrm{A}$ is a rare earth $\mathrm{La}^{3+}, \mathrm{Ba}^{3+}, \mathrm{Pr}^{3+}$ and $\mathrm{B}=\mathrm{Ca}^{2+}, \mathrm{Sr}^{2+}$ ) present a broad variety of phases in which the structural, magnetic and transport properties are intimately linked. The magnetism is the result of a spin interaction due to the overlap of the electronic wave functions of the different atoms. In manganese oxides, where the magnetic Mn ions are separated by oxygen ions, the magnetic exchange is mediated by the overlap of the $2 p$ orbitals of the $\mathrm{O}^{2-}$ ions and the $3 d$ orbitals of the $\mathrm{Mn}^{3+/ 4+}$ ions. The nature of this interaction called super-exchange (SE) depends on the orbitals in plahe SE coupling is in general anti-ferromagnetic. When the electrony. Ts can delocalize over at least two magnetic ions, a ferromagnetic exchange interaction appears called Double Exchange (DE) (see Fig. 10). In doped manganites, this exchange is a mixture of SE and DE because of the different configurations of the electronic orbitals. These two antagonist interactions (ferromagnetic versus antiferromagnetic) can give rise to a magnetic phase separation [10].

The $\operatorname{Pr}_{0.67} \mathrm{Ca}_{0.33} \mathrm{MnO}_{3}$ compound has a ferromagnetic transition below which the application of a magnetic field produces a first order transition from an insulating to a conducting state [11]. In this induced metallic-like state, the magnetization relaxes with time, leading to impressive resistive transitions [12]. This can be understood in a percolation picture where ferromagnetic regions are thermally activated into an antiferromagnetic insulating state. When the last percolation path breaks, the resistivity suddenly jumps to immeasurably large values. In order to finely characterize the phase 


\section{Collection SFN}

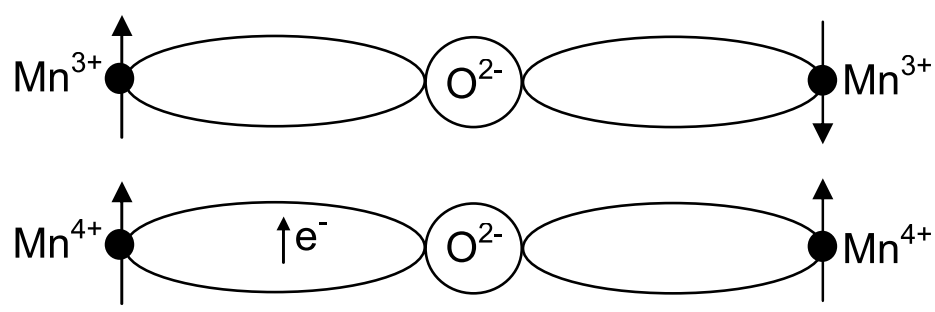

Figure 10. (Top) super-exchange: an anti-ferromagnetic coupling is created by direct overlap of the Mn-O-Mn orbitals. (Bottom) double exchange: an electron is delocalized over the two Mn ions and gives rise to an effective ferromagnetic coupling.

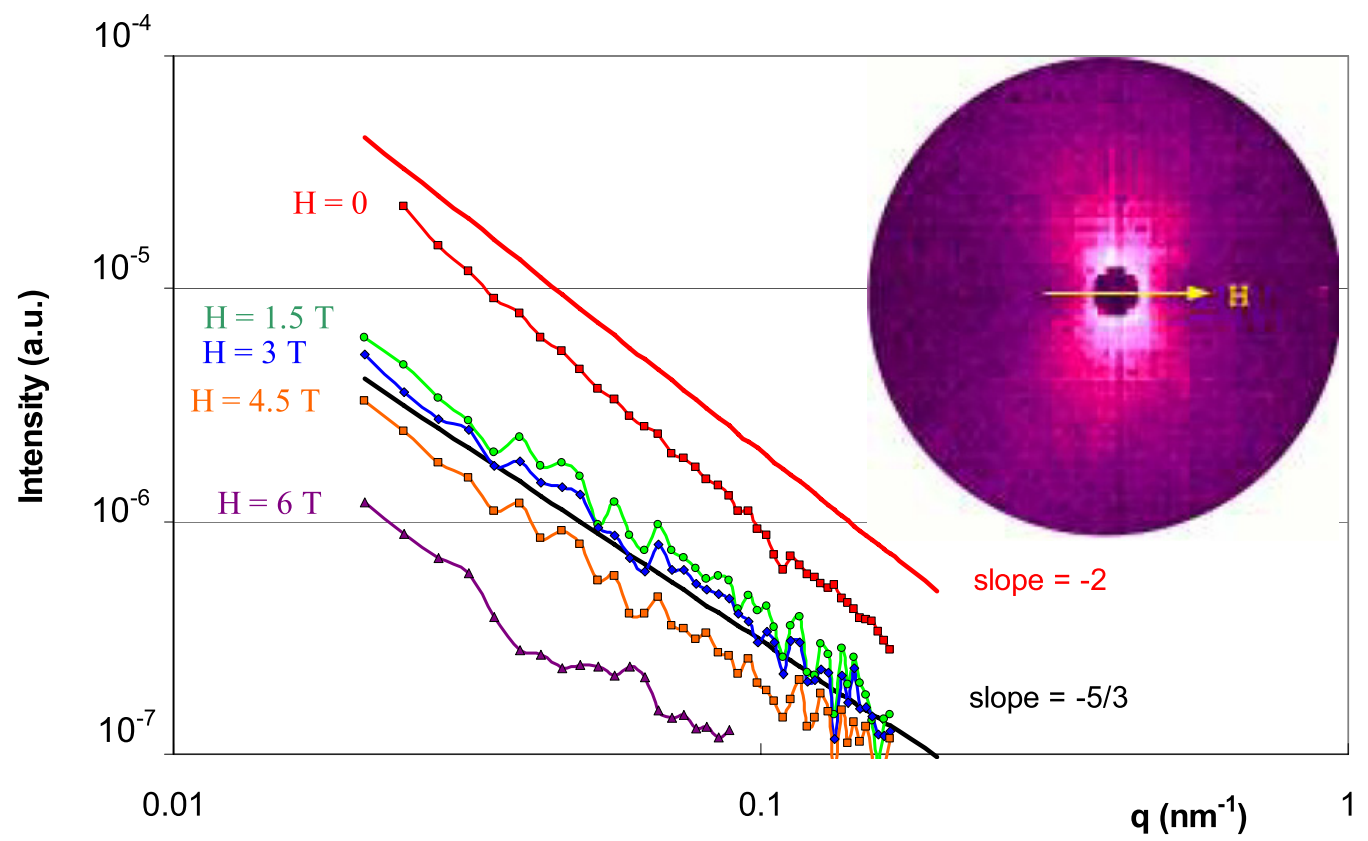

Figure 11. Magnetic SANS intensity from a $\mathrm{Pr}_{0.67} \mathrm{Ca}_{0.33} \mathrm{MnO}_{3}$ single crystal, for a set of increasing fields from 0 to $6 \mathrm{~T}$ after zero field cooling to $4.2 \mathrm{~K}$. The two straight lines are power laws with exponents -2 and $-5 / 3$ (the latter line separates spectra measured in the insulating and metallic states). Inset: typical PSANS 2D spectrum (log scale) showing the $\sin ^{2} \alpha$ contribution of the magnetic scattering (the central black spot is due to the direct beam catcher).

separation in $\operatorname{Pr}_{0.67} \mathrm{Ca}_{0.33} \mathrm{MnO}_{3}$ we have carried out Polarized Small Angle Neutron Scattering (PSANS) under applied fields along with electrical transport and magnetization measurements. The results presented here were obtained on a $1 \times 1 \times 3 \mathrm{~mm}^{3}$ single crystal. The PSANS measurements were carried out at the ORPHEE reactor in Saclay (France) on the spectrometer PAPYRUS.

Unlike bulk measurements which may be interpreted in a phase separation framework because signals do not follow a usual law, the SANS intensity only appears when nanometer size objects are present. Its angular dependence gives the Fourier transform of chemical and magnetic heterogeneities with sizes ranging from $1 \mathrm{~nm}$ to $100 \mathrm{~nm}$. As shown on the typical spectrum in the inset of Figure 11, the SANS signal is characteristic of magnetic scattering with a contribution in $\sin ^{2} \alpha$ with respect to the direction of the applied field. Hence, the scattering entities are purely magnetic. At $4.2 \mathrm{~K}$, the measurements show that the scattering follows a power law $q^{-n}$ with $1.6<n<1.7$ (see Fig. 11). 

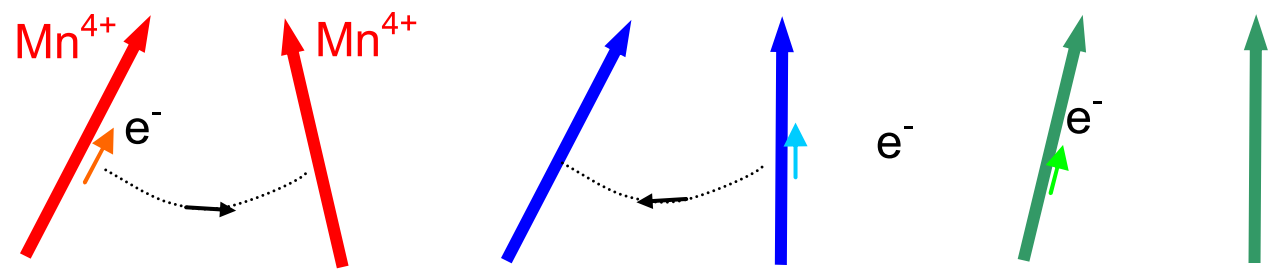

Figure 12. Hopping magnetic exchange: after each electronic hop, the magnetic moments of the Mn ions get better and better aligned.

This fractionnary exponent corresponds to fractal dimensions identical to the one observed in dilute polymer solution $\left(q^{-5 / 3}\right)$. This parallel suggests that the phase separation observed in $\operatorname{Pr}_{0.67} \mathrm{Ca}_{0.33} \mathrm{MnO}_{3}$ could be of filamentary type. In order to validate this hypothesis, we have modeled in a self consistent way the magnetic and transport properties which are intimately linked.

In the $\operatorname{Pr}_{0.67} \mathrm{Ca}_{0.33} \mathrm{MnO}_{3}$ compound, the charge carriers are localized by a random magnetic potential. The electronic conduction takes place by random hopping of the charge carriers from one $\mathrm{Mn}$ ion to a neighboring site. This type of conduction is described by the variable range hopping. We propose a model in which after each electronic hop, the electrons transfer their magnetic moment to the new site and align with the $\mathrm{Mn}^{4+}$ ion magnetic moment. In the process, the total magnetic moment of the electron and the Mn ion is preserved (see Fig. 12).

Because hopping happens preferentially between ions of similar spin direction, the exchange becomes stronger as spins are more closely aligned, which naturally results in a tendency to phase segregate. Indeed, once one Mn has in its vicinity another Mn with parallel spin, the hopping probability between this pair is overwhelmingly large and the ferromagnetic interaction will occur exclusively between these two moments. The remaining surrounding Mn ions interact only via SE. In order to demonstrate that this model leads to a filamentary phase segregation, Michel Viret has carried out Monte Carlo simulations treating transport and magnetism in a self-consistent manner (more details can be found in [13]). When hopping is turned on, magnetic filaments containing many parallel spin carriers with an enhanced mobility appear, as shown in Figure 13.

In the zero field-cooled (ZFC) state at $H=0$, the PSANS intensity can be well fitted with an exponent close to -2 (Fig. 11), i.e. a Debye function. This is consistent with previously published SANS data recorded at zero field and interpreted as an average coherence or a red cabbage structure [15]. Debye functions being reminiscent of polymer melts, this indicates, in our picture, that the magnetic filaments are entangled and do not self-avoid. This is understandable because a $\mathrm{ZFC}$ procedure generates a large density of filaments and a highly resistive state where carriers have to hop further than their nearest neighbors to find states lower in energy. Here, "super-exchange screening" does not work since electrons tunnel over distances longer than the screening length. Hence, Gaussian, entangled, randomly magnetized filaments are generated by a ZFC procedure as shown in Figure 13a. When a field is applied, the filaments with their magnetization parallel to the field grow while others shrink. Within the filaments, mobility is large and carriers proceed by nearest-neighbor-hops, mediating ferromagnetic "hopping exchange". Super-exchange interactions screen the filaments to make them self-avoiding (see Fig. 13b) and the measured power laws are around $-5 / 3$. This exponent remains unchanged as only the global SANS intensity decreases with field, even when the sample resistance drops by orders of magnitude at 3.9 T. The variation in intensity results from a combination of a reduction in magnetic contrast as the background is forced to become more ferromagnetic and an increase in density due to the growth of the filaments. Complementary magnetization measurements (not shown here) allow us to conclude that the volume fraction of the filamentary phase increases monotonically as the field is raised. This naturally leads to a picture of magnetic filaments existing across the entire range of fields and becoming fainter as 


\section{Collection SFN}
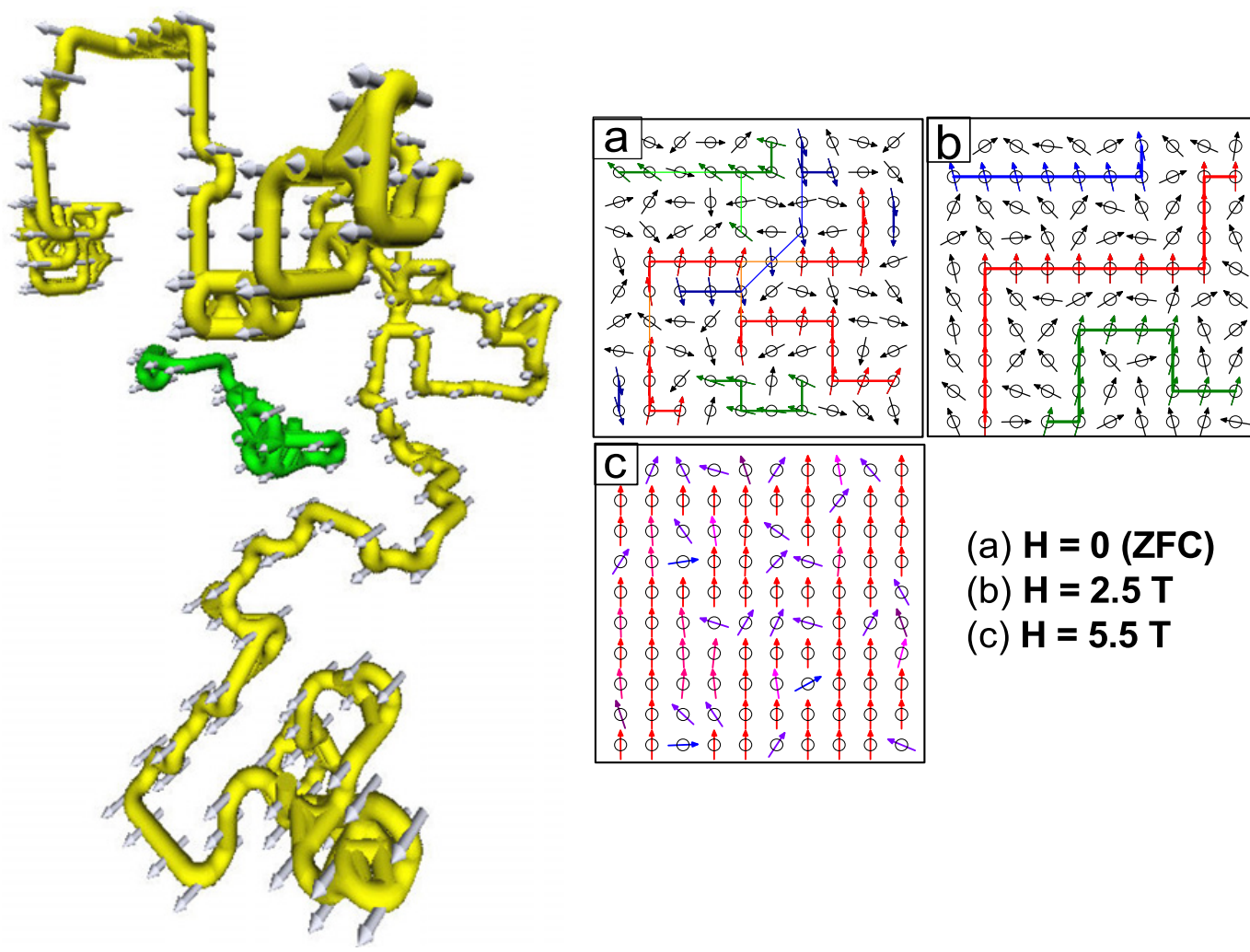

Figure 13. (Left) magnetic filament obtained by Monte-Carlo simulation (calculation performed by Michel Viret). (Right) schematics of the evolution of the filamentary phase with the applied field. From a disordered assembly of small filaments after the ZFC procedure, the applied field makes filaments with spins parallel to $\mathrm{H}$ grow, and shrinks the other one. As the field continues to increase, the filaments percolate at $3.9 \mathrm{~T}$ and then fade into the background when the difference in magnetization is too low to keep the carriers inside the filaments.

the background magnetization increases. At higher fields, the carriers leak out of the filaments into the entire volume (Fig. 13c) which is almost fully magnetized, and produces a homogeneous ferromagnetic phase. The percolation at 3.9 T has no SANS signature since nothing dramatic happens for the magnetic configuration.

In conclusion, we propose here that a ferromagnetic interaction due to electron hopping is responsible for the phase separation in resistive manganites. The random walk motion of the charge carriers leads to the appearance of magnetic filaments which were evidenced by neutron scattering in $\mathrm{Pr}_{0.67} \mathrm{Ca}_{0.33} \mathrm{MnO}_{3}$ single crystals and supported by Monte-Carlo simulations. Further details of this study can be found in [13].

\section{GRAZING INCIDENCE SMALL ANGLE SCATTERING}

\section{Case of thin films and planar nanostructures}

Magnetic surfaces and interfaces at the nanometric scale correspond to very small volumes of matter, of the order of a few micrograms. The use of a grazing incidence geometry increases the neutron interaction with the sample surface and makes neutron scattering experiments feasible. 


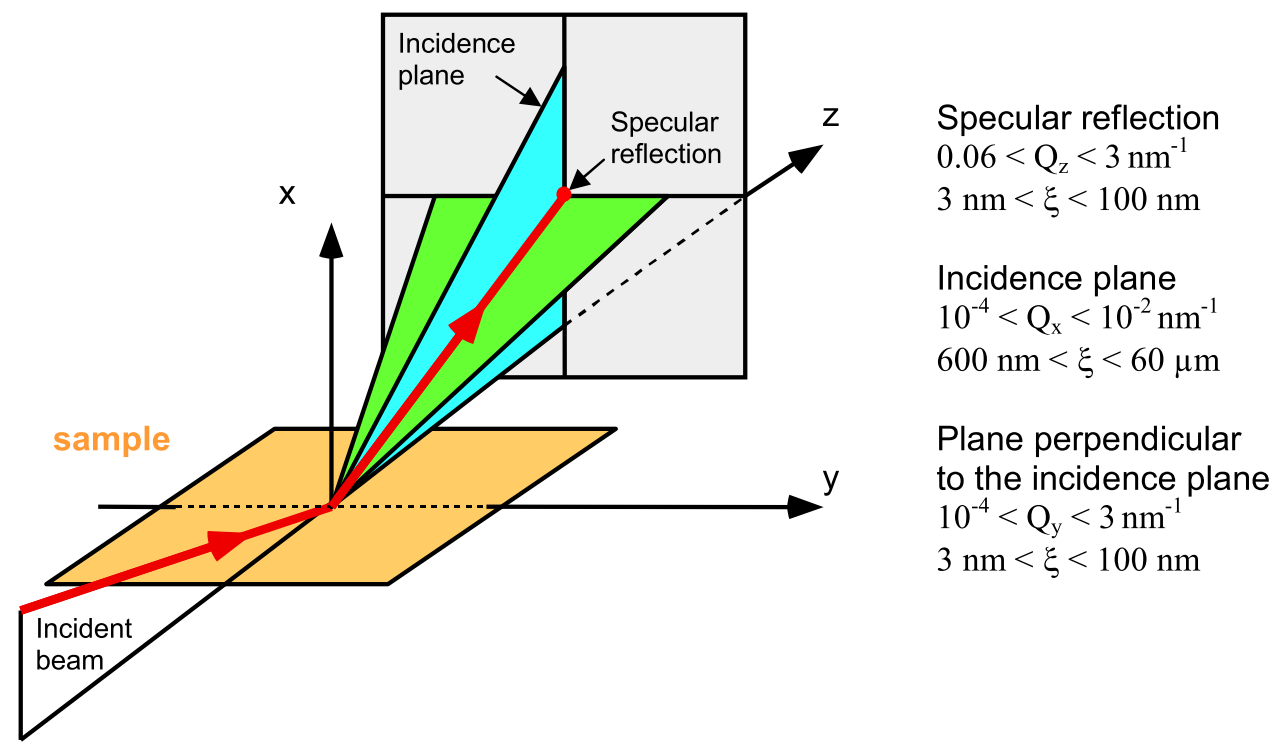

Figure 14. The different surface scattering geometries. (Red line) specular reflectivity geometry; (blue plane) off-specular scattering plane, corresponding to the incidence plane; (green plane) GISANS scattering plane, perpendicular to the incidence plane. These different scattering geometries probe a very wide range of length-scales both in the depth and along the surface of the film.

At grazing incidence, it is possible to distinguish 3 scattering geometries (Fig. 14):

- specular reflection,

- scattering in the incidence plane (off-specular scattering),

- cattering perpendicular to the incidence plane (Grazing Incidence SANS).

These different scattering geometries probe different length-scales $\xi$ and directions in the sample surface. Specular reflectivity probes the structure along the depth in the film $(3 \mathrm{~nm}<\xi<100 \mathrm{~nm})$. Off-specular scattering probes surface features at a micrometric scale $(600 \mathrm{~nm}<\xi<60 \mu \mathrm{m})$. Grazing Incidence SANS probes surface features in the range $3 \mathrm{~nm}<\xi<100 \mathrm{~nm}$. These different scattering geometries allow the study of a very wide range of length-scales $\xi$ ranging from a few nm up to several $\mu \mathrm{m}$ both in the depth and on the surface of the film.

\subsection{Principle}

Since nanosciences are aiming at very small scales (well below $1 \mu \mathrm{m}$ ), off-specular scattering is of limited interest since it can probe only rather large correlation lengths $(\xi>500 \mathrm{~nm})$. This is why surface scattering has been extended to the SANS geometry. In this geometry, one looks at the scattering in the plane perpendicular to the incidence plane (Fig. 14, green plane). The scattering wave vector $Q_{y}$ is in a range comparable to the scattering wave vectors in SANS experiments: $10^{-4}<Q_{y}<3 \mathrm{~nm}^{-1}$. This corresponds to correlation lengths $\xi$ ranging from $3 \mathrm{~nm}$ to $100 \mathrm{~nm}$.

GISANS may typically be used to study small particle sitting on a surface $(\xi \sim 20-100 \mathrm{~nm})$ (Fig. 15a), arrays of nanowires $(\xi \sim 20-100 \mathrm{~nm}$ ) (Fig. 15b), magnetic domains self-organized in a regular structure $(\xi \sim 100 \mathrm{~nm})$ (Fig. 15c), magnetic - structural surface correlations $(\xi \sim 10-20 \mathrm{~nm})$ (Fig. 15d).

The technique of grazing incidence neutron scattering is based on the propagation of an evanescent wave along the surface when the incident angle is smaller than the critical angle of total reflection. 


\section{Collection SFN}
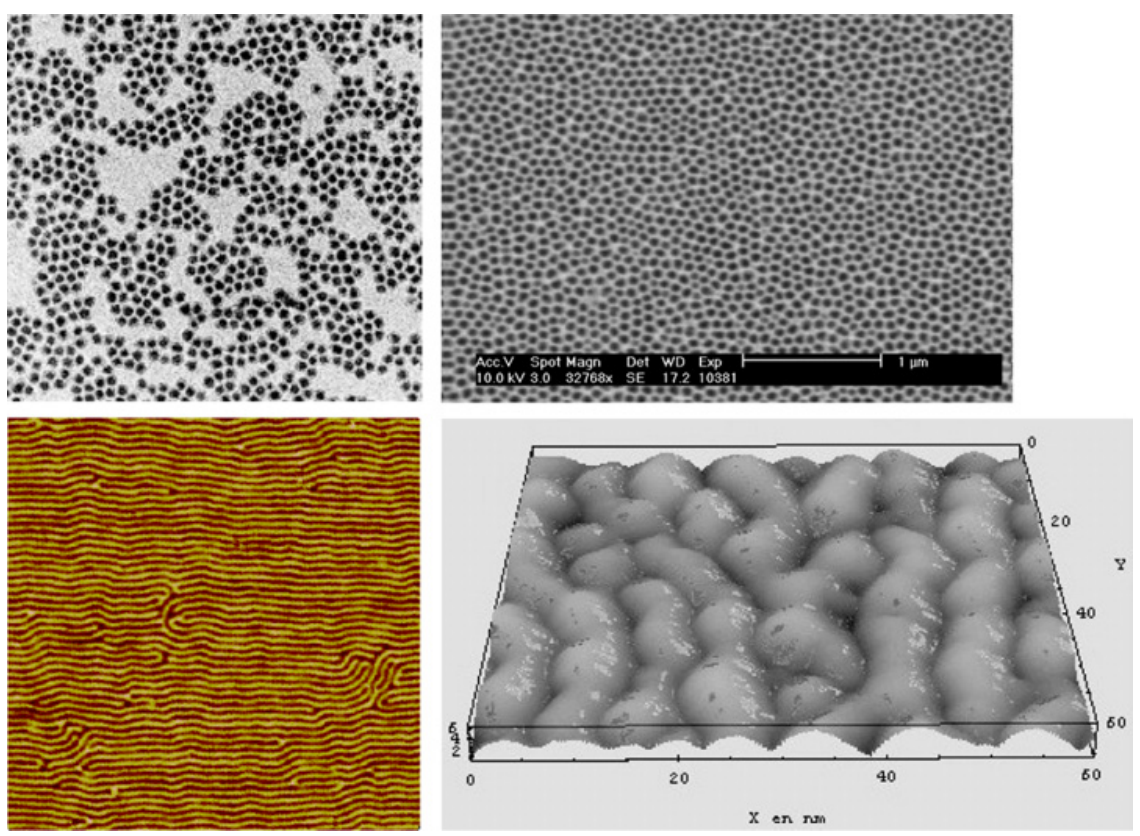

Figure 15. (a) Small particle sitting on a surface; (b) arrays of nanowires; (c) magnetic domains self-organized in a regular structure; (d) magnetic - structural surface correlations.
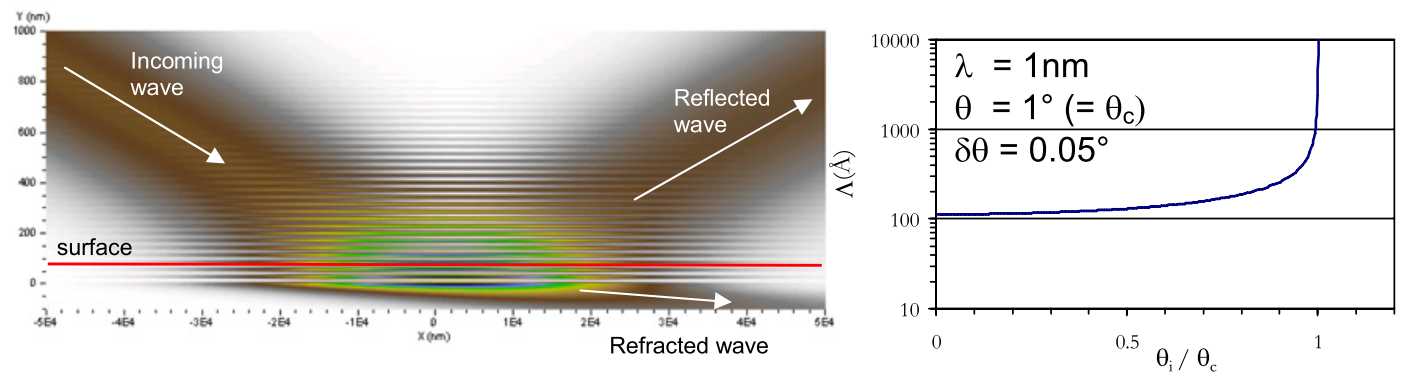

Figure 16. (a) Reflection of a neutron wave on a surface for an incidence angle equal to the critical angle. The plot represent the neutron wave-function density $|\Psi|^{2}$. (b) Penetration depth $\Lambda$ of the neutron in the film as a function of the incidence angle.

Figure 16a illustrates the reflection of a neutron wave incident on a surface for an incidence angle equal to the critical angle of reflection. It can be seen that the neutron wave-function density is increased at the vicinity of the surface. It corresponds to the evanescent wave which travels along the surface. It is possible to calculate the penetration depth of the neutron in the substrate as a function of the incidence angle (Fig. 16b). For very small angles, the neutron remains localized over the top $10 \mathrm{~nm}$ of the surface; as soon as the incidence angle gets close to the critical edge, the neutron wave penetrates deeply in the substrate. When the neutron wave-function is localized at the surface in the form of an evanescent wave, its interaction with the surface and thus the scattering cross section are increased. 

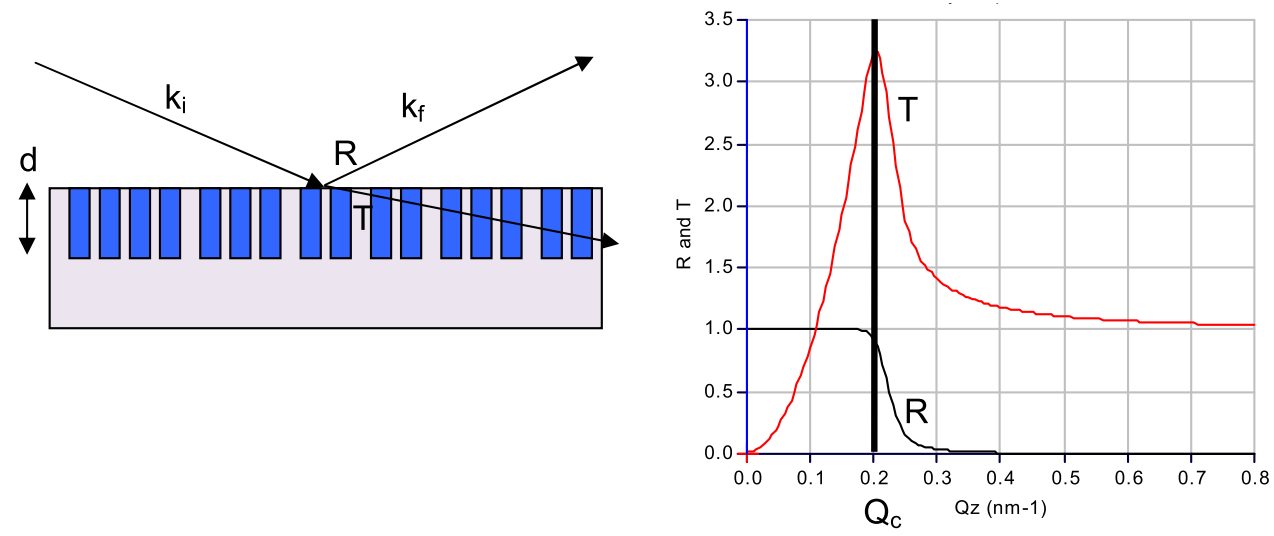

Figure 17. (a) Reflection on buried particles. (b) Enhancement of the transmission coefficients amplitude at the interface close to the critical edge (calculated for an angular and wavelength resolution of $10 \%$ ).

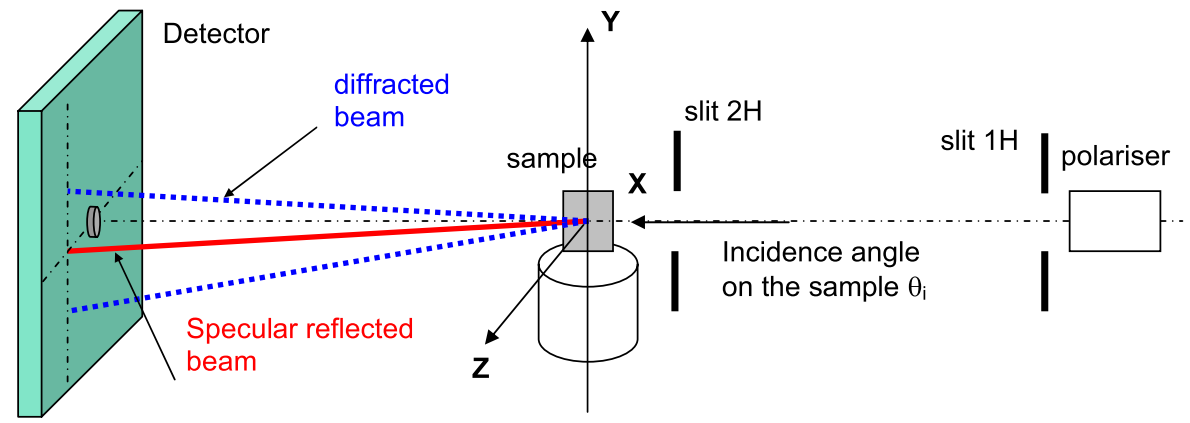

Figure 18. Scheme of the PAPYRUS spectrometer at the LLB.

It can be shown that in the case of buried particles (see Fig. 17a), the scattered intensity can be expressed as [14]:

$$
I(Q) \propto\left|T\left(k_{z}^{i}\right) T\left(k_{z}^{f}\right) F(Q) e^{i Q d}\right|^{2}
$$

where the $T$ factors are the transmission coefficients amplitudes. The amplitude of these coefficient increases very strongly around the critical angle (see Fig. 17b) so that the scattered intensity is enhanced by about a factor 10 .

GISANS experiments are typically performed on SANS spectrometers because a good collimation is required both in the incidence plane (to define the incidence angle) and perpendicular to the incidence plane (to achieve the required resolution for the SANS measurement). The spectrometer PAPYRUS at the LLB has been upgraded so as to make GISANS experiments relatively easy to perform (Fig. 18). It provides a versatile sample environment (Cryomagnet 4K - 6T; Displex 4K ; Electromagnet 1T).

\subsection{Example: Magnetic stripes in FePd and FePt layers}

We present here the first example of a Grazing Incidence SANS experiment on a magnetic thin film [16]. FePt thin film layers self organize themselves in magnetic stripe domains (Fig. 19a). The stripes are almost perfectly ordered in a periodic pattern with a period of about $100 \mathrm{~nm}$. 


\section{Collection SFN}
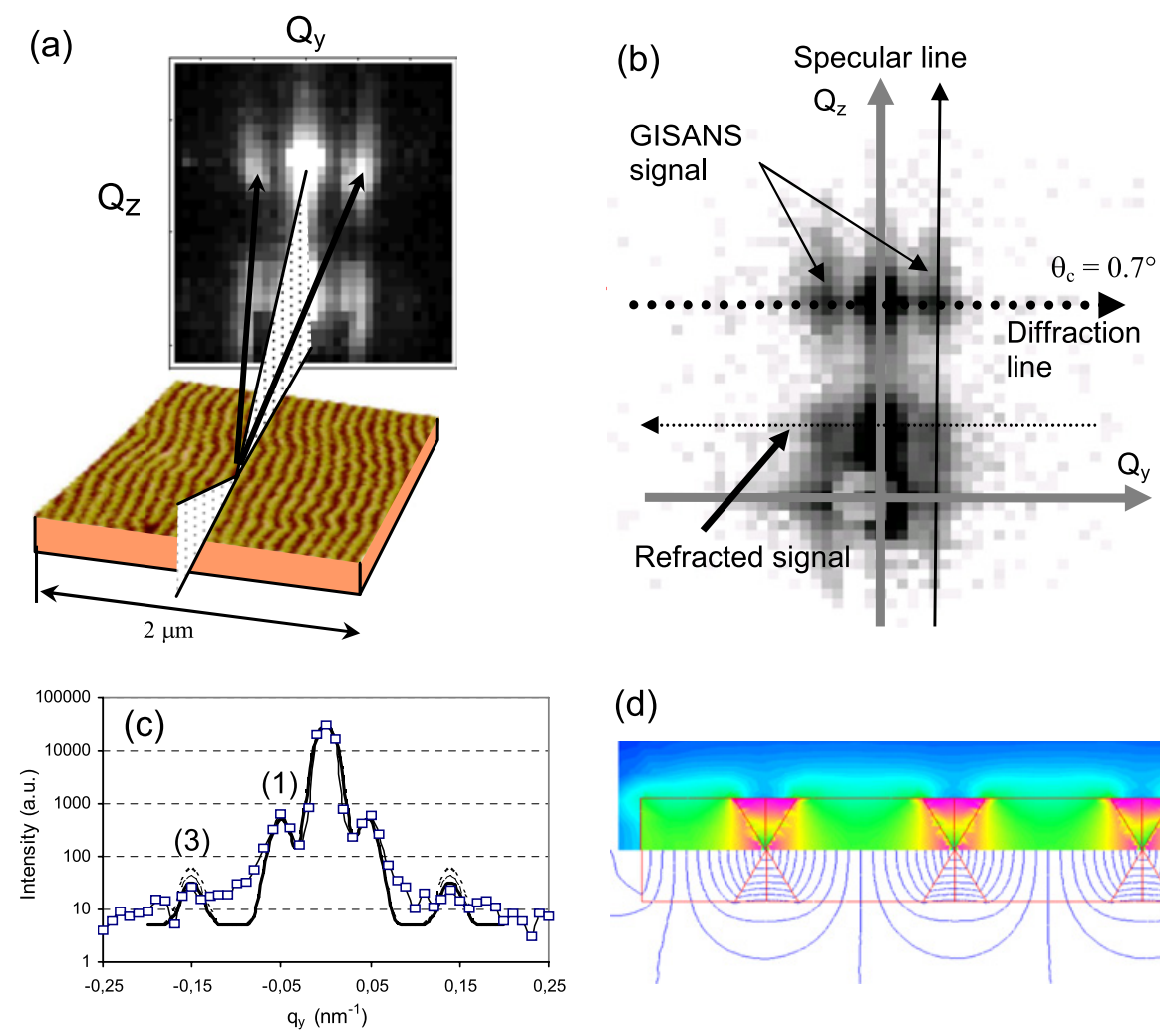

(d)

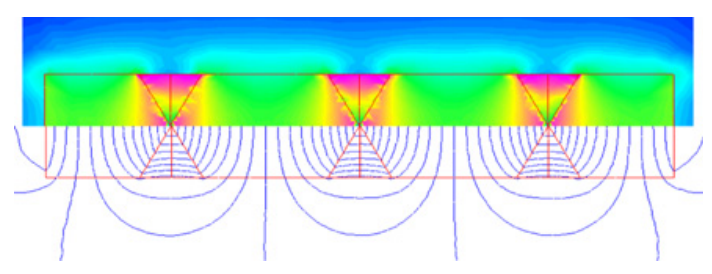

Figure 19. GISANS signal from a magnetic domains nanostructure. (a) Magnetic Force Microscopy image of the magnetic domain and scattering geometry. (b) GISANS signal on the detector for $\theta_{i n}=0.7^{\circ}$. (c) Integrated GISANS signal at constant $q_{z}$ (squares) and fits using different models (lines). (d) Distribution of the magnetic induction in the thin films. The stray field outside the layer are very large and need to be taken into account in the calculation.

Since the system exhibits a magnetic nano-stripe domain structure, it is possible to perform GISANS experiments. The system was thus studied on the GISANS spectrometer PAPYRUS at the LLB. The neutron beam was sent at grazing incidence $\left(\theta_{i n}=0.7^{\circ}\right)$ on the layer, the magnetic domains being parallel to the incidence plane (Fig. 19a). Diffraction from the magnetic domains can be observed. Figure 19b details the different contributions of the Grazing Incidence SANS signal. An integration at fixed $q_{z}$ has been performed and is presented on Figure 19c. Three diffraction orders can be observed. Note that only odd modes can be observed because the magnetic potential is an even function in the plane of the layer. Mode 2 is extinct. In order to quantitatively model the system, we have shown that it is necessary to take into account both the Néel caps between the magnetic stripes as well as the magnetic stray fields (Fig. 20).

A detailed discussion of this experiment can be found in [17].

\section{CONCLUSION}

Neutron scattering can be applied to the study of magnetic nano-objects with lengthscales in the range ( $\xi \sim 3-300 \mathrm{~nm}$ ). A wide range of techniques can be applied to study nanosystems (see Fig. 21).

One may use SANS or reflectivity techniques or a combination of them. The strong magnetic scattering allows for quantitative magnetic studies. The strong contrast between $\mathrm{H}$ and $\mathrm{D}$ allows 


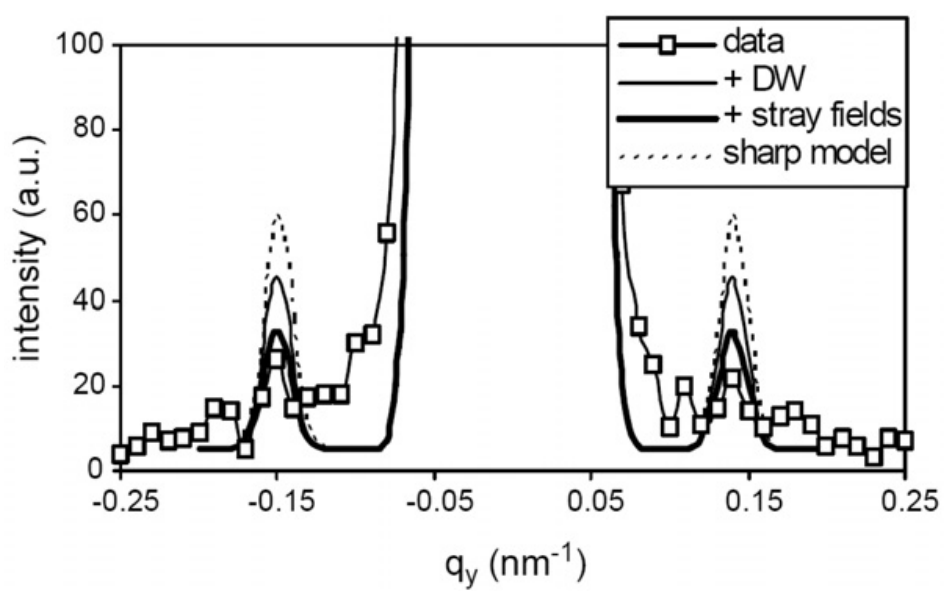

Figure 20. Cut along the $Q_{y}$ direction for $\theta_{i n}=0.7^{\circ}$; experimental points (squares) and simulations for a step function (sharp domain walls model), using the simulated micro-magnetic structure with domain walls (+DW), taking into account the stray fields outside the layer (+stray fields). The intensity ratio between the first and third order can only be accounted for by take into account the stray fields.

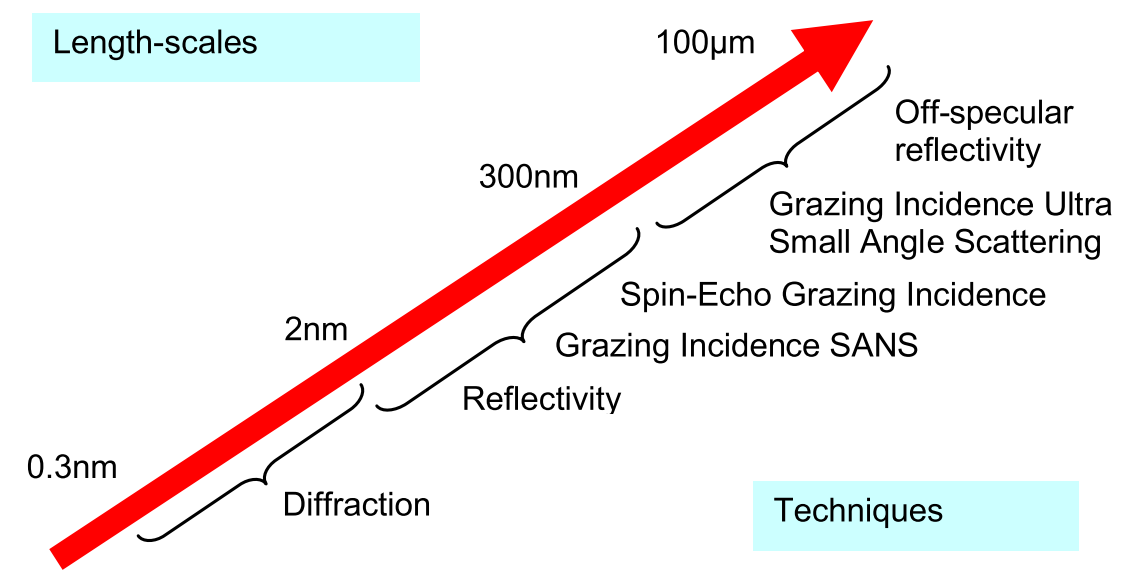

Figure 21. Correlation lengths and suitable neutron scattering techniques.

performing contrast variation measurements which enable unraveling the details of the organic matter organization.

Neutron scattering also allows setting up experiments in extreme conditions (temperature, magnetic field, pressure).

\section{References}

[1] C. Desvaux, C. Amiens, P. Fejes et al. Nature Materials 4, 750-753 (2005).

[2] D. Ung, Y. Soumare, N. Chakroune, G. Viau, M.J. Vaulay, V. Richard, F. Fievet, Chem. Mater. 19, 2084-2094 (2007).

[3] O. Fruchart, P.O. Jubert, C. Meyer et al., Journal of Magnetism and Magnetic Materials 239 (2002) 224-227. 


\section{Collection SFN}

[4] C.P. Li, I.V. Roshchin, X. Batlle et al., J. Appl. Phys. 100, 074318 (2006).

[5] A. Wiedenmann et al., J. Phys. 18 (2006) s2713.

[6] M. Klokkenburg et al., Phys. Rev. E 75 (2007) 51408.

[7] M. Pannetier, T.D. Doan, F. Ott, S. Berger, N. Persat, C. Fermon, Europhysics Letters 64, 524-8 (2003).

[8] M. Viret, H. Glattli, C. Fermon et al., Euro. Phys. Lett. 42, 301-306 (1998).

[9] A. Pautrat, J. Scola, C. Simon et al., Phys. Rev. B 71, 064517 (2005).

[10] E. Dagotto, Nanoscale phase separation and colossal magnetoresistance: the physics of manganites and related compounds (Springer-Verlag, Berlin, 2003).

[11] Y. Tomioka, A. Asamitsu, H. Kuwahara, Y. Morimoto and Y. Tokura, J. Phys. Soc. Jpn. 64, 3626 (1995).

[12] A. Anane et al., Phys. Rev. B 59, 77 (1999).

[13] M. Viret et al., Phys. Rev. Lett. 93 (2004) 217402.

[14] Rauscher et al., Phys. Rev. B 52 (1995) 16855.

[15] Ch. Simon, S. Mercone, N. Guiblin, C. Martin, A. Brulet and G. Andre, Phys. Rev. Lett. 89, 2072021 (2002).

[16] C. Fermon et al., Physica B 267-268 162-7 (1999).

[17] M. Pannetier, F. Ott, C. Fermon, Y. Samson, Physica B 335, 54-8 (2003). 